Revue d'histoire de l'Amérique française

ZRS REVUE D.HISTOIRE DE L'AMÉRIQUE FRANÇAISE

\title{
Plaintes contre l'administration de la justice (1807) (suite)
}

\section{Jean-Pierre Wallot}

Volume 20, numéro 2, septembre 1966

URI : https://id.erudit.org/iderudit/302570ar

DOI : https://doi.org/10.7202/302570ar

Aller au sommaire du numéro

Éditeur(s)

Institut d'histoire de l'Amérique française

ISSN

0035-2357 (imprimé)

1492-1383 (numérique)

Découvrir la revue

Citer cet article

Wallot, J.-P. (1966). Plaintes contre l'administration de la justice (1807) (suite). Revue d'histoire de l'Amérique française, 20(2), 281-290.

https://doi.org/10.7202/302570ar d'utilisation que vous pouvez consulter en ligne.

https://apropos.erudit.org/fr/usagers/politique-dutilisation/ 


\section{PLAINTES CONTRE L'ADMINISTRATION DE LA JUSTICE (1807) (suite)}

Panet venait de décocher à l'avocat général et aux juges qu'il ne suffisait pas de lire les lois du Canada, "y ayant dans les lois, les mœurs et une tradition d'usage, une science que des étrangers n'ont pas de les bien comprendre et appliquer". Poursuivant sa plaidoirie, il martela autorités et arrêts du Conseil qui interdisaient aux notaires de déplacer leurs "minutes", sauf lorsqu'on contestait l'authenticité même d'un testament. Planté jouissait done du droit incontestable de garder chez lui l'original du testament. Bien plus, la Cour n'avait, par aucune loi, le choix de le lui ôter, de le lui laisser, ou de le mettre au greffe". Par conséquent, la troisième question s'effondrait d'elle-même. Si les juges pouvaient tripoter à leur gré les lois et la jurisprudence, ils seraient "souverains".

L'avocat général Sewell ne s'embarrassa pas de tant de légalité. Il balaya les lois et les auteurs cités: valables, ils ne l'étaient que pour la période précédant la Conquête. “... il s'agissait de savoir jusqu'à quel point la jurisprudence avait été changée depuis". ${ }^{2}$ Le droit romain et la coutume prescrivaient l'ouverture d'un testament cacheté par un juge. Celui-ci devait ensuite le remettre à un notaire. Or, depuis la Conquête, les greffiers ou protonotaires étaient sur le même pied que les

1 Voir notre Revue, XIX: 551-560; XX: 28-43.

2 En d'autres termes, les entorses aux lois françaises, depuis la Conquête, servaient à excuser celles qu'on s'apprêtait à perpétrer ! Quoi qu'il en soit, Sewell ne manquait pas de suite dans les idées. Il tirait les mêmes conclusions, dans l'ordre religieux. Ainsi, lors de l'audition de la cause Lavergne vs Bertrand, en 1805, il plaida que la Conquête avait aboli le poste d'évêque catholique et cédé au roi tous les pouvoirs de cette charge: "... there is no Catholic Bishop of Quebec by law. His office became extinct at the conquest..." (Cité dans R. Christie, History of Lower Canada... (6 vols., Québec, 1848-1855), 6: 91.) 
notaires à cet égard. En outre, la loi de 1801 avait validé la manière anglaise de prouver les testaments. Or, celui de Duchesnay se conformait à la forme anglaise sur certains points, v.g. quant aux biens meubles. Les juges pouvaient donc les considérer sous la forme anglaise. De plus, il contenait des substitutions en faveur de personnes non nées, que la cour devait protéger. Enfin, l'intérêt public nécessitait le dépôt des testaments dans les archives publiques: depuis 1801, un père disposait d'une grande liberté de tester $;^{3}$ et chez les notaires, les testaments étaient sujets au feu, au vol, etc.

Pierre Bédard, avocat mais aussi chef du parti canadien, persifla à propos des arguments "frivoles" de son adversaire Sewell. Selon la théorie du procureur général, on "pouvait actuellement hasarder toutes sortes d'opinions" pour la période après la Conquête. Comment faire équivaloir les greffiers aux notaires, sauf que le mot notaire était inséré dans "PROTONOTAIRES", la nouvelle appellation des greffiers? L'Acte de Québec permettait de tester selon la forme anglaise ou la française. Et parce qu'une partie seulement du testament suivait par hasard la première, fallait-il ignorer toutes les autres sections conformes à la forme anglaise et incompatibles avec l'anglaise? "Nous aurons une espèce d'amphibie, dont la moitié sera dans la forme anglaise \& moitié dans la forme française; ou bien il faudra... en sacrifier la moitié pour avoir la satisfaction de faire juger l'autre dans la forme anglaise." En ce

3 La loi de 1801 clarifia l'Acte de Québec à propos de la liberté testamentaire. En effet, le statut impérial de 1774, apparemment clair, avait donné lieu en fait à de nombreuses difficultés d'interprétation. Par la loi coloniale de 1801, le testateur "est investi d'une liberté presque totale de répartir à sa mort ses biens - à l'exception de ceux spécialement affectés aux douaires de l'époux survivant et des enfants - comme bon lui semblait entre les membres de sa famille. Bien plus, cette liberté s'étendait même hors du cercle restreint de la famille légitime, car toutes les entraves coutumières étaient subitement levées." (André Morel, Les Limites de la liberté testamentaire dans le droit civil de la province de Québec (Paris, 1960), 40. Voir particulièrement les pages 17 à 45.) Le professeur Morel analyse les difficultés engendrées par l'article X de l'Acte de Québec, puis la portée de la loi de 1801. Ainsi, il explique comment ce dernier statut, introduit par des juristes de formation française et dans des termes du droit français, transplantait un principe de droit anglais dans le corps du système juridique français préexistant. 
qui avait trait à la nécessité de rendre le testament public à cause des substitutions, une "expédition" suffisait; ou bien, l'on ne conservait l'original que le temps requis à cet effet. Par ailleurs, l'usage avait modifié le droit romain, quant à l'ouverture de certains testaments par un juge. Les héritiers euxmêmes avaient assisté et consenti à l'ouverture du testament de Duchesnay par le notaire. Enfin, outre la loi, l'utilité publique exigeait le dépôt chez les notaires: déplacements et frais réduits au minimum; assurance des habitants de toujours trouver leurs titres au même endroit; etc.

Bédard se buta à plusieurs interruptions du juge en chef. À un moment, celui-ci clama sèchement que "cet office [celui de notaire] était bien connu". Bédard le corrigea: on confondait trop facilement les notaires d'ici, investis de pouvoirs très étendus, et les "notaires publics" d'Angleterre, sortes de "huissiers". Ici, forcer un notaire à comparaître avec ses minutes, sauf dans le cas d'un "faux", c'était aussi extraordinaire que de convoquer un juge d'une cour inférieure avec ses registres. Concrètement, la "nouvelle manière de déposer les testaments" résulterait en de nombreux inconvénients: obligation pour les habitants de se rendre dans les villes, frais accrus, etc. Le juge s'interposa à nouveau. Il avait apparemment déjà tranché la question. Il trouvait donc trop longue une argumentation qui contredisait son avis préconçu.

Le lendemain, 11 juin, le juge en chef promulgua la décision de la cour: le testament demeurerait au greffe. “.... la Cour, se défendait-il, ne décidait pas sur tous les testaments olographes, mais... sur celui-ci seulement parce qu'il contenait des substitutions". La séance dégénéra ensuite en une véritable comédie vaudevillesque. Bédard s'enquit si la cour publierait l'original ou la copie du testament, optant quant à lui pour la copie. Planté voulut ouvrir la bouche. Le juge en chef se fâcha. Le notaire risqua quand même qu'il valait mieux utiliser la copie, puisque "l'original" ne pouvait se trouver à plusieurs endroits à la fois! Le juge Williams n'y voyait goutte: il ne pouvait comprendre comment un testament "olographe" pou- 
vait être un "original". Bédard l'affirma tel, puisqu'on l'avait confié au notaire afin d'en tirer des "expéditions". Pendant que tous se colletaient, le juge de Bonne devait savourer la scène, d'autant plus que c'étaient maintenant ses ennemis politiques qui "dansaient". Bédard eut beau invoquer les lois, qualifier la procédure de la cour d'“illégale \& irrégulière", rien n'y fit. Le jugement fut lu, puis déposé dans "l'office" de la Cour et enregistré.

JEAN-PIERRE WALLOT, Musée national, Ottawa.

\section{LE CANADIEN, 4 JUILLET 1807}

Dépôt du Testament du Mr. Duchesnay, \&c. (Continuation du Plaidoyer de Mr. Panet).

Enfin M. Panet a lu plusieurs citations dont M. Planté avait délivré copie à la Cour. Elles étaient tirées du Parfait Notaire, de Furgole sur les testaments que le Juge en pays de droit écrit ou même le Notaire peut ouvrir. Denisart verbo minute page 349 , où il établit que les Notaires ne doivent déplacer les minutes que dans le cas où, après avoir été arguées de faux,il leur a été ordonné par un jugement exprès. Denisart encore, verbo scellé, page 468 , nom. 66 , dit que "C'est le Notaire qui doit être dépositaire de la minute et non le Greffier", il cite l'arrêt du Conseil du 3 déc. 1688, ordonnant au Lieutenant de la Prevôté qui s'était, lors des scellés, emparé d'un testament, de le remettre à Me Beaudry, Notaire, au Châtelet. Autre arrêt du 27 mars 1737, Denisart, aux nombres 68 et 60 explique le sens de l'acte de Notoriété pour Paris, cite une sentence du Châtelet conforme aux Arrêts et Ordonnances y énoncés, portant que les Notaires sont obligés de se charger du Dépôt des testaments, même qu'un Notaire qui s'était saisi irrégulièrement d'un testament lors des scellés requis, serait tenu de se charger par Dépôt du même testament et que ce ne serait qu'à son refus seulement qu'un autre Notaire l'aurait en dépôt, Pothier, traité des donations testamentaires, ch. 1, parag. 3, som. 6, page 300 , en parlant des formes extrinsèques des testaments olographes, dit: $1^{\circ}$ l'acte de dépôt doit s'en faire chez un Notaire. Pigeau, procédure du Châtelet de Paris, tom. 2 , pages 282 et 283 , pose le vrai cas d'un testament clos, trouvé lors des scellés requis, et qu'il doit être en tous cas mis en dépôt "entre les mains du Notaire nommé pour l'inventaire. Répertoire de Jurisprudence, tom. 12, verbo Notaires, page 203, 1re colonne, c'est aux Notaires à recevoir les dépôts des testaments "privativement à tous autres officiers", selon les maximes et arrêts qu'il cite en grand nombre. D'après tant d'autorités, Me. Panet a dit que la conclusion contre la 2e question proposée, était certainement que Mr. Planté, comme Notaire public, avait le droit de garder l'original du Testament en Dépôt, et que la Cour n'avait, par aucune loi, le choix de le lui ôter, de le lui laisser, ou de le mettre au greffe.

Sur la 3e et dernière Question.

Et si la Cour a ce choix, ne serait-il pas de l'intérêt public qu'elle préférât de le mettre au greffe? 
Mr. Panet a dit que cette question était aussi oiseuse que la seconde, parce que la solution de la 1re, était que le Notaire a droit de garder le dépôt du testament. Cette $3 \mathrm{e}$ question devient non seulement inutile en Cour de Justice, mais encore spéculative en matière de législation. Quand la Coutume écrite et l'Ordonnance prononcent les droits et qualités des vraies parties ou officiers publics, que personne ne les conteste régulièrement et que la jurisprudence des anciens et nouveaux arrêts ont jugé les cas semblables à celui dont il s'agit, on peut dire selon les maximes de Jurisprudence et comme dit Mr. Ferrière en son Dictionnaire de Droit, verbo "Lois que les Juges sont obligés de suivre". $1^{\circ}$. Les Ordonnances faites et non celles à faire; $2^{\circ}$. Les coutumes écrites et non celles à écrire, et verbo Notoriété, où il établit que "les Juges ne peuvent pas seuls donner de ces sortes d'actes", et il dit que si tous les officiers et intéressés n'y ont pas procédé, il dépendrait des Juges, qui ne sont pas souverains, de changer à leur gré les lois et les usages de leur jurisdiction, et de se rendre par ce moyen les maîtres de la jurisprudence. Mr. Planté, Notaire, en ce cas n'aurait jamais dû être taxé en Cour de jalousie contre le Greffier, pas même des prétentions de l'Archevêque de Canterbery, sur les formes des testaments en Angleterre telles que celles que les anciens Evêques en France ont très antiquement exigées et perdues. Aucune partie publique ni privée n'a régulièrement procédé contre Me Planté, ainsi il faut passer sous silence tout ce que spéculativement on pourrait dire en faveur des dépôts de testaments chez les Notaires qui sont les Officiers publics et qui de droit et de fait méritent le choix et la confiance des sujets de sa Majesté dans cette Province.

Mr. l'Avocat Général prit ensuite la parole du côté opposé et admit que toutes les autorités citées par Monsieur Panet étaient exactes, que tout ce qu'il avait dit était conforme à l'ancienne jurisprudence, et qu'il aurait eu raison avant la conquête, mais qu'il s'agissait de savoir jusqu'à quel point la jurisprudence avait été changée depuis. Qu'il avait été fait tant de changements depuis la conquête, qu'il était souvent bien difficile de savoir jusqu'où s'étendaient les effets de ces changements.

Qu'il était question de savoir s'il était à l'option des Juges de déposer les minutes d'un testament olographe chez un Notaire ou chez un Greffier. Que toutes les autorités qui avaient été citées avaient leurs sources dans le Droit Romain et étaient tirés du Digeste, livre 29, titre 3, et qu'il n'y avait en France aucune loi, ni aucun usage particulier sur cette matière. Il cita l'acte de Notoriété du Châtelet de Paris de 1708, qui était fondé sur le Droit Romain et observa que lorsqu'il survenait des doutes sur quelque point de Jurisprudence en France, on assemblait les gens du Roi pour avoir leurs opinions et qu'on en dressait des actes de notoriété; que suivant celui qu'il citait, lorsqu'un testament olographe était trouvé cacheté, c'était le Juge qui en faisait l'ouverture, et qu'il le déposait ensuite chez un Officier Public. "L'usage du Châtelet a pour fondement la disposition de ces lois (des lois Romaines); et en conformité d'icelle, le Juge doit ouvrir le testament cacheté en présence de celui qui l'apporte, et le déposer à l'instant entre les mains de l'Officier Public", actes de Notoriété du Châtelet par Denisard, p. 338. L'Avocat Général remarque sur les termes Officier Public, que c'était un Notaire entre les mains duquel le Juge déposait le testament; mais que par les changements qui avaient eu lieu ici, les Greffiers, c'est-à-dire, les Prothonotaires, étaient sur le même pied que les Notaires à cet égard. Il cita la note sur ce passage en ces termes: "Mr. le Lieutenant Civil est dans l'usage de parapher les testaments et d'en constater l'état, quand on les lui apporte. Il fait à ce sujet un Procès Verbal, sur lequel il rend une Ordonnance, portant que la 
minute du testament (s'il est olographe) sera déposée chez un Notaire qu'il nomme et qui s'en charge en conséquence". - L'Avocat du Roi dit ensuite, que l'acte de la 14e année de sa Majesté (bill de Québec) avait introduit une manière particulière de tester, qui n'était point connue ni autorisée par les anciennes lois; il cita la 10e section qui est en ces termes: "Pourvu aussi qu'il sera et pourra être loisible à toute et chaque personne, propriétaire de tous immeubles, meubles ou intérêts, dans ladite Province, qui aura le droit d'aliéner lesdits immeubles, meubles ou intérêts, pendant sa vie, par ventes, donation ou autrement, de les tester et léguer à sa mort par testament et acte de dernière volonté, nonobstant toutes lois, usages et coutumes à ce contraire, qui ont prévalues, ou qui prévalent présentement en ladite Province, soit que tel testament soit dressé suivant les lois du Canada, ou suivant les formes prescrites par les lois d'Angleterre. Qu'en conséquence de cette clause il s'était introduit une nouvelle manière de prouver les testaments, savoir de les prouver devant les Juges, comme on le fait dans les cours de Probates en Angleterre.

Qu'à cet effet on avait, pendant un temps, donné aux Juges de la Cour des Plaidoyers communs par une clause de leur commission, les pouvoirs de la Cour de Probate. Qu'on avait ensuite discontinué de mettre cette clause dans leur commission et qu'ils avaient toujours continué de recevoir les preuves des testaments olographes jusqu'en 1801. Que la législature ayant alors pris cette matière en considération, il s'éleva un doute sur le pouvoir des Juges et que ce doute fut levé par la 2de section du Statut de cette Province de la 41 e année de sa Majesté, chap. 4 qui porte: "Et comme il s'est élevé des doutes sur la manière actuelle de prouver les testaments faits et dressés suivant la forme Anglaise, devant un ou plusieurs des Juges des Cours de Jurisdiction Civile en cette Province, qu'il soit de plus statué, que telle preuve vaudra et aura force de la même manière que si elle était faite devant une Cour de Probate". - Que le testament de Monsieur Duchesnay était un bon testament dans la forme Anglaise, quant aux meubles, parce que par les lois d'Angleterre, il n'était pas nécessaire qu'un testament fut signé par des témoins pour valoir quant aux meubles: que la signature de trois témoins n'était nécessaire que pour les testaments d'immeubles. Et qu'ainsi le testament de Mr. Duchesnay étant également dans la forme Anglaise et Française, les Juges avaient le droit d'en recevoir la preuve, comme d'un testament fait dans la forme Anglaise. - Qu'en second lieu, ce testament contenait des substitutions et que pour cette raison la loi exigeait pour sa validité qu'il fut publié en Jugement, et il cita l'Ordonnance de Moulins de 1567, et dit que ces substitutions étaient en faveur de personnes qui n'étaient pas EN ESSE (pas nées) et n'avaient d'autres protecteurs que la Cour, et que la Cour pour satisfaire sa conscience avait le droit de se saisir du testament et de le garder en sa possession, afin d'en assurer la publication. - Que la Cour pouvait faire cela légalement, parce que le dépốt avait été irrégulièrement fait chez le Notaire. - Que, suivant l'acte de Notoriété déjà cité, le testament trouvé cacheté devait être ouvert par le Juge. Que dans le cas présent le Notaire avait pris sur lui "de faire une chose qu'il n'avait pas droit de faire" le dépôt était illégal, qu'il n'y avait point de dépôt, et que le Notaire n'était pas en droit de réclamer le privilège. Car, dit-il, qu'aurait fait le Juge si on lui eût porté le testament pour en faire l'ouverture? Il aurait ordonné qu'il fût mis dans les records ou archives publiques.

Il dit qu'il était de l'intérêt public que les testaments y fussent mis; que les nouveaux Statuts avaient agrandi le pouvoir de tester; qu'un père pouvait actuellement déshériter totalement ses enfants; ce qu'il ne pouvait 
faire auparavant, et que les enfants devenant par là plus intéressés à supprimer les testaments, la défiance devait devenir plus grande.

Qu'un testament déposé dans les voûtes de cette maison était bien plus en sûreté contre les accidents du feu, que dans les études des Notaires. - Que, lorsqu'un testament serait déposé dans cette maison, il y aurait deux chances, parce que outre l'original déposé il y aurait une copie dans les régistres. - Q'il y avait beaucoup plus de facilité à faire la preuve du testament et que les frais seraient bien moindres. - Que si l'on supposait qu'il y eût plusieurs actions à poursuivre, que quelqu'un des créanciers fit une inscription de faux, à quelles dépenses cela ne donnerait-il pas lieu ? - Qu'il espérait donc que la Cour n'adopterait pas la manière proposée de déposer le testament; que si l'on ne déclairait pas le Probate avec effet, ce serait s'écarter de la loi positive qui exigeait un enregistrement.

Mr. Bédard en réplique à l'Avocat Général dit: Que la manière sérieuse et assurée avec laquelle l'Avocat Général avait entrepris sa tâche lui avait d'abord causé quelque crainte, mais qu'il avait été beaucoup rassuré à mesure que ses arguments avaient été déployés; qu'ils lui avaient tous parus frivoles, et qu'il croyait qu'ils avaient eu besoin de toute la rapidité de son élocution pour paraître tolérables.

Que l'Avocat Général avait d'abord admis que toutes les citations qui avaient été faites en faveur de M. Planté étaient justes et qu'il aurait eu raison avant la conquête, mais qu'on pouvait actuellement hazarder toutes sortes d'opinions et que c'était en conséquence qu'il avait hazardé celle qu'il venait de soutenir. - Que l'Avocat Général avait avancé que les Greffiers étaient actuellement sur le même pied que les Notaires, quant $a u$ droit d'avoir les testaments en dépôt, mais qu'il n'avait pu distinguer quelle preuve il en avait donné. Q Qu'il supposait que c'était parce que le mot de NOTAIRE entre actuellement dans la composition du titre des Greffiers qui par leurs nouvelles commissions sont devenus PROTHONOTAIRES. - Que l'Avocat Général fondait son premier argument sur ce que le Statut de la 14e année de sa Majesté avait étendu la liberté de tester et qu'il avait introduit une nouvelle forme de faire les testaments, et sur ce que le Statut de la 41e année de sa Majesté avait confirmé les Juges dans l'usage où ils avaient été de faire les Probates des testaments dans cette nouvelle forme; mais que le Statut de la 14e année de sa Majesté avait laissé la liberté de faire les testaments dans l'ancienne forme Française. Que les Juges n'avaient jamais été dans l'usage de faire les Probates des testaments faits dans cette forme, et que le Statut de la 41e année ne leur confirmait que le pouvoir de faire les probates des testaments dans la forme Anglaise; ce dernier Statut porte: "Et comme il s'est élevé des doutes sur la manière actuelle de prouver les testaments" faits et dressés suivant la forme Anglaise" devant un ou plusieurs des Juges, \&c. qu'il soit de plus statué, que telle preuve vaudra et aura force de la même manière que si elle était faite devant une Cour de Probate". Qu'ainsi tout était resté tel qu'il était quant à la forme Française.

Qu'il était vrai que l'Avocat Général avait prétendu que le testament de Monsr. Duchesnay devait être regardé comme un testament fait dans la forme Anglaise, parce qu'il se trouverait bon en Angleterre quant aux meubles, mais que c'était une singulière manière de prouver qu'un testament est dans la forme Anglaise parce qu'il se trouve par hasard qu'une partie de ce testament serait bonne dans cette forme. - Le Testament de Monsr. Duchesnay contient aussi la disposition des immeubles de Mr. Duchesnay. Cette partie sera nulle si on la considère dans la forme Anglaise. Dans quelle forme l'Avocat Général voudra-t-il qu'on la con- 
sidère ? Ce sera apparemment dans la forme Française et nous aurons une espèce d'amphibie, dont moitié sera dans la forme Anglaise et moitié dans la forme Française; ou bien il faudra que le testament de Mr. Duchesnay soit regardé comme nul quant aux immeubles, et en sacrifier la moitié pour avoir la satisfaction de faire juger l'autre dans la forme Anglaise. Que ce serait avoir bien peu d'égard pour l'intention évidente du Testateur et bien mal suivre l'intention de l'acte qui donne le choix de deux formes, que de choisir celle où le testament ne serait bon que pour moitié.

Que le 2d argument de l'Avocat Général était que le testament contenait des substitutions qui exigeaient la formalité de la publication. Qu'il était bien vrai que le testament contenait des substitutions, et bien vrai aussi qu'il était nécessaire pour cette raison qu'il fut publié, mais qu'il y avait une grande distance de là à la question actuelle et que l'Avocat Général n'avait pas montré comment, de la nécessité de cette publication, s'ensuivait la nécessité d'ôter le testament au Notaire.

Que ce n'était pas une chose nouvelle que de voir des actes sujets à insinuation ou à publication; que tous les jours les Notaires faisaient des donations sujettes à insinuation, mais que ce n'était pas une raison d'aller chercher le Notaire avec sa minute et de les amener en Cour pour remplir cette formalité; que cette publication n'était pas le sujet pour lequel ce testament avait été apporté en cette Cour; que personne même ne la demandait; qu'en supposant que la Cour crût de son devoir d'y faire procéder, ce ne pourrait être une raison de garder le testament parce que ce n'était pas la minute qu'on publiait, mais une expédition, et que quand la Cour croirait de son devoir de faire publier la minute même, ce ne serait pas encore une raison de garder le testament plus longtemps que jusqu'à ce que la publication fût faite. Que le vrai moyen de parvenir à cette publication, régulièrement, était de remettre le testament à Mr. Planté afin qu'il en délivrât une expédition pour la publier. Qu'en supposant que ce testament eût dû être ouvert par un juge, il ne s'ensuivrait pas que la Cour dût actuellement l'ôter au Notaire pour le punir de l'avoir ouvert lui-même; que dans tous les cas où le Juge faisait l'ouverture, il devait déposer le testament "chez un Notaire"; que tous les auteurs et les arrêts constatent ce point. Que Furgole est le seul qui dise que le Juge a le choix de la déposer au Greffe ou chez un notaire; qu'il ne donne aucune raison de cela, ne cite aucun exemple où le cas ait jamais eu lieu; que c'était une de ces idées vagues, telle qu'il en échappe souvent aux auteurs, et qu'il était impossible que le seul Furgole l'emportât sur tous les autres auteurs et sur les arrêts; qu'ainsi en supposant que le Juge eût fait l'ouverture, il aurait dû à l'instant ordonner que le testament fût déposé chez un Notaire, mais qu'il n'y avait aucune nécessité que le testament fût ouvert par les Juges, que cela pouvait avoir été pratiqué en France, mais qu'aucune loi ne l'avait exigé; que c'était un simple usage qui avait eu lieu à l'imitation du droit Romain; mais que cet usage avait été changé, puisque Furgole dit lui-même tom. ler, page 98, "comme l'usage a introduit de faire les subscriptions des testaments devant Notaire, on a introduit aussi la coutume de faire procéder à leur ouverture et publication devant Notaire, et ces sortes de publications sont aussi valables que celles devant le Juge. No. 12. Les témoins testamentaires étant en présence du Juge ou le Notaire, le testament doit leur être exhibé, \&c. No. 13. Le Juge ou Notaire doit dresser procès verbal, \&c. Page 101, Quand une fois le testament a été enregistré ou au Greffe du Juge ou "dans les registres du Notaire qui en a fait l'ouverture, la transcription qui en a été faite a la même autorité que l'original, \&c.". 


\section{LE CANADIEN, LE 11 JUILLET 1807}

Dépôt du Testament de Mr. DUCHESNAY, \&c.

Mr. Bédard, en continuation de sa réplique, dit: qu'il y avait lieu à l'ouverture des testaments par le Juge dans le cas de l'apposition des scellés lorsque les héritiers sont absents; mais que quand les héritiers étaient présents, eux qui seuls peuvent avoir des intérêts contraires au testament, leur consentement était la meilleure formalité pour le testament, et que personne n'avait le droit de le contester quand ils l'admettaient eux-mêmes. Que c'était les héritiers qui avaient appelé Mr. Planté et lui avaient donné le testament en dépôt et qu'ils avaient le droit que ce testament y restât déposé; qu'un tel dépôt, une fois fait, était sacré, que c'était le droit et le devoir du Notaire de le garder; que personne ne pouvait le dispenser de ce devoir, et qu'il était inouï qu'un Notaire eût jamais été dépossédé d'un dépôt de cette nature.

Qu'en admettant contre toutes les autorités que le Juge qui fait l'ouverture d'un testament eût le choix de le déposer au greffe ou chez un Notaire, et que ce fût actuellement le moment de faire le choix, il devrait être fait en faveur du Notaire. Que le public avait intérêt que les testaments fussent déposés chez les Notaires plutôt qu'au greffe; parce qu'au greffe, ils devenaient publics et sujets à l'inspection de tout le monde et qu'un testateur pouvait avoir des raisons que sa dernière volonté ne fût pas ainsi livrée à la curiosité du public.

Que l'Avocat Général pour prouver que les greffiers méritent la préférence avait eu recours à la solidité des voûtes, qu'il était vrai que les voûtes étaient bien solides, mais ... Le Juge en Chef dit qu'il n'était pas question des voûtes, mais seulement de la loi; Mr. Bédard dit qu'il ne parlerait donc pas des voûtes ni des accidents du feu; mais qu'il considérerait le mérite des Notaires et des Greffiers, faisant abstraction des voûtes et de la manière dont étaient bâties les maisons - Qu'on devait juger de la confiance que méritaient les Notaires et les Greffiers, dans le cas présent, par celle que la loi mettait dans leurs offices; que l'office des Notaires était aux yeux de la loi un office d'une bien plus grande importance et qui méritait beaucoup plus de confiance que celui des Greffiers. Que les Notaires étaient dépositaires de tous les titres qui assureraient les fortunes des sujets de sa Majesté, et que la loi ne confiait aux Greffiers que les papiers qui concernent les biens en litige qui sont déjà risqués dans la procédure. - Qu'il était ridicule de témoigner du scrupule de confier le testament en question à un office auquel la loi avait déjà confié les titres de toutes les fortunes; que c'était surtout une précaution outrée que de prétendre mieux choisir que les parties intéressées elles-mêmes, qui avaient confié le testament en question au Notaire et qui voulaient qu'il le gardât; que l'acte du dépôt fait chez Mr. Planté avait été cité dans tous les actes de la succession, et que les intéressés futurs chercheraient en vain ce testament dans les minutes de Mr. Planté; que si on se permettait ainsi de transporter d'un lieu à un autre les minutes des Notaires, ce serait ôter au public cette certitude qu'il a de trouver dans le même lieu les actes qui assurent les propriétés; que l'on sentait combien cette certitude contribuait à donner cette assurance que les propriétaires doivent avoir de leurs propriétés; que l'on sentait combien cette assurance diminuerait si on avait l'idée qu'il serait permis de faire voyager les Notaires avec leurs minutes; qu'il fallait n'avoir pas d'idée de l'office des Notaires pour avoir de pareilles prétentions. - Le Juge en Chef dit que cet office était bien connu. - Mr. Bédard dit que s'il était bien connu, il ne l'avait pas toujours été, comme on le voyait par le titre de Notaires publics qu'on avait donné 
aux Notaires; que ce titre, que les Notaires avaient encore, montrait qu'on avait ignoré entièrement l'importance et la nature de cet office qu'on avait confondu avec celui des NOTAIRES PUBLICS d'Angleterre qui est infiniment inférieur à celui des Notaires de ce pays et qui n'a aucune analogie avec lui; l'emploi de Notaire public étant de courir çà et là protester les lettres de change, office que les Notaires peuvent remplir ici, mais qui est proprement du ressort des huissiers. Qu'avec des idées pareilles, il n'était pas surprenant qu'on n'ait pas regardé l'office des Notaires avec l'égard qu'il mérite par son importance. Qu'il n'y avait que dans certains cas tel que celui d'inscription de faux et quelqu'autres nommés par la loi, où il était permis de transporter les minutes des Notaires. Qu'il ne savait pas quelle idée on avait eu des Notaires, mais que c'était pour lui une chose bien extraordinaire que de voir paraître un Notaire dans la boête aux témoins avec sa minute sous son bras; que c'était suivant lui une chose aussi extraordinaire qu'il le serait de voir amener dans cette boête les Juges des cours inférieures avec les Registres de leurs cours; que les minutes des Notaires étaient de vrais records dans l'idée qu'on attachait à ce mot dans les lois Anglaises, et qu'il était aussi impossible de faire voyager les uns que les autres, excepté dans les cas nommés par la loi et avec les formalités requises.

Que l'Avocat Général avait détaillé les commodités qui résulteraient si le testament en question était déposé dans l'office du greffe, particulièrement dans le cas d'inscription de faux; mais qu'il n'avait pas montré les incommodités qui résulteraient plutôt de cette nouvelle manière de déposer les testaments, en ce qu'elle obligerait les gens de la campagne qui sont éloignés du greffe de s'y transporter pour y déposer les testaments. Que les inscriptions de faux n'auraient pas plus souvent lieu à l'avenir qu'ils ne l'avaient eu jusqu'à présent; mais que les dépôts de testaments étaient très fréquents, et occasionneraient beaucoup de voyages et de frais aux parties. - Que Mr. L'Avocat Général avait dit que tout se faisait au greffe avec promptitude et à bon marché; Mr. Bédard dit que pour le bon marché il en doutait... Le Juge en Chef témoigna que l'argument était trop long. Mr. Bédard s'excusa sur ce qu'il ne faisait que répondre aux arguments de l'Avocat Général et cessa de parler. - Mr. Planté remit à la Cour copie des autorités qui ne sont que citées dans les plaidoyers, et la Cour mettant en délibéré, fit entrer sur le registre ce qui suit:

Sur requête de Madame Duchesnay, et consorts.

Mr. A. Panet, Conseil de Mtre Planté a ouvert le cas.

Mr. l'Avocat Général a été ouï sur la question publique.

M. P. Bédard a répliqué à l'Avocat Général de la part de Me. Planté. Dont acte. La Cour mit en délibéré. 